\title{
A critical appraisal of paliperidone long-acting injection in the treatment of schizoaffective disorder
}

This article was published in the following Dove Press journal:

Therapeutics and Clinical Risk Management

27 January 2016

Number of times this article has been viewed

\section{Pierre Chue' \\ James Chue ${ }^{2}$}

'Department of Psychiatry, University of Alberta, ${ }^{2}$ Clinical Trials and

Research Program, Edmonton, AB,

Canada

Video abstract

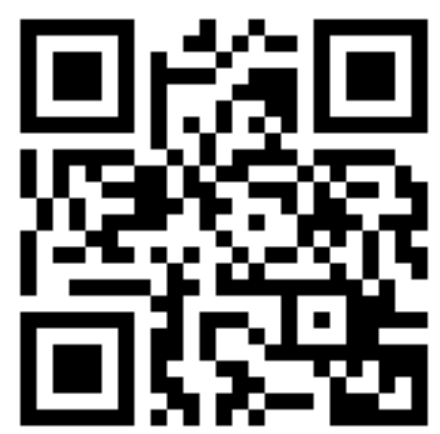

Point your SmartPhone at the code above. If you have a QR code reader the video abstract will appear. Or use

http://youtu.be/RX-IAEm7fSU

Correspondence: Pierre Chue Third Floor, 9942-108 Street, Edmonton, AB, Canada T5K 2J5

Tel +I 7803427944

Fax +17804259317

Email pchue@ualberta.ca
Abstract: Schizoaffective disorder (SCA) is a chronic and disabling mental illness that presents with mixed symptoms of schizophrenia and affective disorders. SCA is recognized as a discrete disorder, but with greater heterogeneity and symptom overlap, leading to difficulty and delay in diagnosis. Although the overall prognosis is intermediate between schizophrenia and mood disorders, SCA is associated with higher rates of suicide and hospitalization than schizophrenia. No treatment guidelines exist for SCA, and treatment is frequently complex, involving offlabel use and polypharmacy (typically combinations of antipsychotics, mood stabilizers, and antidepressants). Oral paliperidone extended-release was the first agent to be approved for the treatment of SCA. As in schizophrenia and bipolar disorder, adherence to oral medications is poor, further contributing to suboptimal outcomes. The use of an antipsychotic in a long-acting injection (LAI) addresses adherence issues, thus potentially reducing relapse. Paliperidone palmitate represents the LAI formulation of paliperidone. In a long-term, double-blind, randomized, controlled trial of adult patients ( $\mathrm{n}=334$; intent-to-treat [ITT]) with SCA, paliperidone long-acting injection (PLAI) significantly delayed risk of relapse compared to placebo (hazard ratio 2.49, 95\% confidence interval, $1.55-3.99 ; P<0.001)$. This study demonstrated the efficacy and safety of PLAI when used as either monotherapy or adjunctive therapy for the maintenance treatment of SCA. The results are consistent with a similarly designed study conducted in patients with schizophrenia, which suggests a benefit in the long-term control of not only psychotic but also affective symptoms. No new safety signals were observed. When used in monotherapy, PLAI simplifies treatment by reducing complex pharmacotherapy and obviating the necessity for daily oral medications. PLAI is the second agent, and the first LAI, to be approved for the treatment of SCA; as an LAI formulation, there is the advantage of improved adherence and simplified treatment in the long-term management of SCA.

Keywords: second-generation antipsychotics, paliperidone palmitate, long-acting injection, schizoaffective disorder

\section{Introduction}

Schizoaffective disorder (SCA) represents a chronic and disabling mental illness that presents with mixed symptoms of schizophrenia and affective disorders. ${ }^{1}$ Historically, SCA has posed a challenge from a diagnostic perspective given that both psychotic and affective symptoms can occur either concurrently or at different time points throughout the course of the illness leading to poor diagnostic reliability and validity. ${ }^{2-4}$ Thus, a substantial revision between Diagnostic and Statistical Manual of Mental Disorders, fifth edition (DSM-5) and previous versions included the requirement that mood symptoms be assessed for the entire course of the illness and that mood symptoms meeting criteria for a major mood episode must be present for the majority 
of the total duration of the active and residual portion of the illness. ${ }^{5}$ There is a paucity of studies in SCA and even fewer encompassing current DSM-5 criteria. The difficulty in diagnosis contributes to the complexity of management and likely to a delay in the implementation of appropriate treatment for many patients. ${ }^{6}$ In contrast to schizophrenia and mood disorders, there are no accepted treatment guidelines, and until relatively recently, there were no medications specifically approved for the treatment of SCA. The present article reviews the current medications that have received a formal indication for the disorder and focuses on the published data for paliperidone long-acting injection (PLAI).

\section{Schizoaffective disorder}

The prevalence of SCA is approximately one-third that of schizophrenia with a similar onset in early adulthood, but typically a delayed diagnosis, which likely contributes to the prognosis that is intermediate between schizophrenia and affective disorders. ${ }^{7-10}$ There is a high rate of comorbidity, particularly with substance use and anxiety disorders and, similar to schizophrenia and bipolar disorder, life expectancy in patients with SCA is decreased compared with the general population. ${ }^{11}$ While, approximately one-third of patients presenting in outpatient settings have a diagnosis of SCA, for those needing inpatient care, SCA and schizophrenia are equally represented. ${ }^{12,13}$ Thus, individuals with SCA are potentially more disabled by their illness and, consequently, utilize a greater share of health care resources, particularly inpatient care, which is the costliest. Furthermore, the rates of substance use, hospitalization, and suicide are greater than are observed in schizophrenia, with the risk of suicidality being correlated with depressive symptoms. ${ }^{14-16}$ Insight is impaired, though generally to a lesser degree than in schizophrenia, and there is less cognitive deficit. ${ }^{17,18}$ Compared with schizophrenia, negative symptoms and social avoidance are less frequent, but depressive and guilt symptoms occur more commonly. ${ }^{19}$ Social and occupational dysfunction is common, but somewhat more variable than in schizophrenia. ${ }^{20,21}$ Quality of life is decreased compared to the general population and correlates with affective symptoms, particularly depressive. ${ }^{22,23}$ Individuals with SCA appear more at risk of a mood episode if affective symptoms persist after a psychotic episode.

Recent studies suggest some differences in genetic risk $^{24-26}$ and neurobiology in SCA versus other psychiatric disorders. ${ }^{27-30}$ Individuals with SCA are younger and more likely to be Caucasian, compared with individuals with schizophrenia. ${ }^{31}$ Overall, there is a greater incidence of SCA in females compared with males, which has been related to the fact that the depressed subtype occurs more frequently in females. ${ }^{32}$

\section{Pharmacotherapy of SCA}

The pharmacotherapy of SCA is complex and typically requires long-term treatment with mood stabilizers, antipsychotics, and antidepressants, often in combination to control psychotic and affective symptoms. ${ }^{31-37}$ Patients with SCA are more likely to be receiving concomitant psychotropic medications than patients with schizophrenia. ${ }^{31}$ Long-term adherence to such complex regimens is poor, leading to suboptimal outcomes. ${ }^{38-41}$ There are very few studies evaluating adherence rates, specifically in SCA versus schizophrenia or bipolar disorder, but prevalence rates of nonadherence have been reported in the range of $41 \%$ to $44 \%{ }^{38,39}$ Poorer adherence is reported in the context of psychotic symptoms, manic relapses, personality disorder comorbidity, and lithium therapy.

Antipsychotics have been commonly used in the treatment of SCA with rates in monotherapy ranging from $34 \%$ to $55 \%$, and rates in combination with mood stabilizers and/or antidepressants ranging from $23 \%$ to $87 \%$. ${ }^{11,31,35,36} \mathrm{Up}$ to $93 \%$ of patients with SCA receive an antipsychotic despite the fact that currently there are no widely accepted guidelines for the treatment of SCA. ${ }^{42}$ There are very few randomized controlled trials with respect to the use of first-generation antipsychotics (FGAs) and none for FGA-long-acting injections (LAIs), specific to SCA. In fact, the evidence concerning efficacy of antipsychotics in SCA is largely extrapolated from studies in schizophrenia incorporating patients with "related psychotic disorders", including SCA, the latter often representing too small a sample from which to draw any robust conclusions. ${ }^{33,42}$ The second-generation antipsychotics (SGAs) have demonstrated efficacy in the treatment of affective disorders, including bipolar and major depressive disorders, as well as in the treatment of schizophrenia. A review of the pharmacologic treatment of SCA and schizophrenia with mood symptoms suggested that SGAs could be more effective than FGAs. ${ }^{37}$ Furthermore, evidence of benefit of the SGAs in the treatment of SCA was demonstrated in studies with oral risperidone. ${ }^{43,44}$ Finally, a systematic review of antipsychotic switching in SCA discussed switching to oral SGAs for primarily tolerability reasons, and to risperidone LAI in situations where adherence may be an issue. ${ }^{45}$ Risperidone LAI is the only LAI to be approved for the treatment of bipolar disorder as well as schizophrenia. ${ }^{46}$ In a 6-month, open-label study of SCA 
patients $(\mathrm{n}=249)$, treatment with RLAI significantly improved mean scores for the Positive and Negative Syndrome Scale (PANSS) (total and subscales) and Global Assessment of Functioning (all $P<0.001) .{ }^{47}$ A long-term, open-label study with olanzapine LAI in patients with schizophrenia $(n=931)$ reported maintenance of effect as measured on the PANSS, although there were no specific data on the subset of SCA patients $(2.4 \%){ }^{48}$

Paliperidone is the 9-OH metabolite of risperidone and shares with the parent molecule, a similar but not identical, CNS neurotransmitter receptor profile. ${ }^{49}$ Following prior approval for the treatment of schizophrenia, paliperidone extended-release (ER) was approved in 2009 (USA) for the acute treatment of SCA as monotherapy and as an adjunct to mood stabilizers and/or antidepressants in adults. ${ }^{50-53}$ Similarly, following prior approval for the treatment of schizophrenia, PLAI received approval in 2013 (USA) for use in adults for the acute treatment of SCA as monotherapy and as an adjunct to mood stabilizers and/or antidepressants. ${ }^{54}$ At this time, neither any other antipsychotics, nor indeed, any agents are formally approved for the treatment of SCA.

By virtue of their formulation and, hence, parenteral administration, the LAI antipsychotics address the partial adherence inherent to all oral antipsychotics. Consequently, the LAIs have been shown to reduce the risk of relapse in patients with schizophrenia and, thus, potentially improve outcomes. ${ }^{55}$ The administration of an antipsychotic LAI results in more prolonged and relatively constant plasma concentrations of the active moiety providing longer term efficacy and better tolerability, particularly for treatment emergent adverse effects (TEAEs) related to peak serum levels. To date, there have been no studies of FGA-LAIs, specifically in SCA. Two recent expert consensus guidelines recommend the use of LAIs in the management of SCA, with specific consideration of an SGA-LAI as the first-line strategy. ${ }^{56,57}$ The development of an SGA-LAI for the treatment of SCA represents a logical step for an illness characterized by both psychotic and affective symptoms and is associated with poor adherence to oral therapies and consequent risk of relapse.

\section{Pharmacology of PLAI}

Paliperidone is the major active metabolite of risperidone produced through alicyclic hydroxylation at position 9, primarily by cytochrome P450 2D6, and to a much lesser extent by CYP3A4.

As with the parent molecule, the therapeutic effects of paliperidone are thought to be primarily mediated by its profile of antagonism at $\mathrm{D}_{2}$ and $5-\mathrm{HT}_{2 \mathrm{~A}}$ receptors. ${ }^{49}$ However, the $5-\mathrm{HT}_{2 \mathrm{~A}} / \mathrm{D}_{2}$ binding ratio for paliperidone is significantly higher than for risperidone, and it also possesses a lower affinity for $\alpha_{1}$ and $\alpha_{2}$ adrenergic receptors and higher affinity for $5-\mathrm{HT}_{7}$ receptors. ${ }^{58,59}$ Furthermore, paliperidone affects expression levels and phosphorylation of complex I and V proteins in synaptoneurosomal preparations of rat prefrontal cortex in a similar way to mood stabilizers, such as lithium and valproate, in contrast to risperidone. These differential effects on mitochondrial movement, protein expression, phosphorylation, and cellular signaling pathways have only recently been recognized and are partly attributed to the presence of the hydroxyl group, which results in increased hydrophilicity compared with risperidone. ${ }^{60}$

PLAI is an aqueous suspension of paliperidone palmitate nanocrystals resulting in a sustained release profile over a 4-week period following intramuscular injection. ${ }^{61,62}$ After intramuscular injection, there is initial slow dissolution because of low aqueous solubility followed by more rapid hydrolysis to paliperidone, which is then systemically absorbed, but drug release begins from day 1 and continues for up to 126 days (median $T_{\max }$ of 13 days). The pharmacokinetics of PLAI are characterized by a one-compartment disposition model with zero-/first-order absorption. The two initial doses of PLAI are given as deltoid injections, thereby achieving therapeutic concentrations of paliperidone within 7 days, and do not need oral supplementation. This is because of a $C_{\max }$ greater by $28 \%$ compared with gluteal injections. After the two initial deltoid injections, PLAI can be administered as either a deltoid or a gluteal injection with comparable $T_{\max }$ and cumulative exposure after four injections for the $100 \mathrm{mg}$ dose, and the mean steady-state peak-to-trough ratio is 2.2 and 1.8 , respectively.

\section{Efficacy of paliperidone in SCA}

The efficacy of oral paliperidone in the treatment of SCA was first demonstrated in two, 6-week, international, double-blind, placebo-controlled studies with paliperidone ER conducted in adults (18-65 years), incorporating either flexible-dose (3-12 mg/d; $n=304$; intent-to-treat [ITT]) or fixed-dose (low dose 3-6 mg/d or high dose $9-12 \mathrm{mg} / \mathrm{d}$; $\mathrm{n}=310$; ITT) design. ${ }^{63,64}$ In these studies, $79.5 \%$ and $66.9 \%$ of subjects presented with prominent manic and depressive symptoms, respectively, while $46.4 \%$ had mixed symptoms; $45 \%$ of subjects were receiving adjunctive mood stabilizers and/or antidepressants. These registration studies demonstrated that oral paliperidone improved psychotic and mood symptoms in SCA and was effective as monotherapy or as an adjunct to mood stabilizers and/or antidepressants. 
The efficacy of paliperidone in a long-acting, injectable formulation in SCA was subsequently demonstrated in an international, randomized, double-blind, placebo-controlled study conducted in adults (18-65 years). ${ }^{65}$ This was a registration study that led to the approval of PLAI as not only monotherapy, but also adjunctive therapy for SCA. ${ }^{54}$ Although the study was conducted using DSM-IV diagnostic criteria for SCA, the authors reported that following the release of DSM-5, investigator assessments demonstrated that $95 \%$ of randomized subjects also met the current diagnostic criteria; first-episode subjects were excluded to ensure diagnostic homogeneity. The Clinical Global Impression of Severity for Schizoaffective Disorder (CGI-S-SCA) scale was utilized in this study. It evaluates the four domains of SCA (positive, negative, manic, and depressive) and was previously developed and validated in studies of SCA; good correlations between the CGI-SCA domains and corresponding symptom-based scale measures of the PANSS (positive and negative subscales), Young Mania Rating Scale (YMRS), and Hamilton Depression Rating Scale (HDRS-21) have also been demonstrated. ${ }^{63,64}$

The study comprised three phases: an initial screening phase; a 25-week (13-week, flexible-dose lead-in and a 12-week, fixed-dose stabilization), open-label phase; and a 15-month, randomized, double-blind, relapse-prevention phase (PLAI, n=170; placebo, $n=164$; ITT). Relapse and impending relapse were defined, a priori, as the first occurrence of any one of the following criteria:

- Psychiatric hospitalization

- Intervention to avoid hospitalization (increased level of care or additional psychotropic medications)

- Suicidal or homicidal ideation or behavior

- Increase in a PANSS item score $\geq 6$ (if score $\leq 4$ at randomization) for P1 (delusions), P2 (conceptual disorganization), P3 (hallucinatory behavior), P4 (excitement), P6 (suspiciousness/persecution), P7 (hostility), G4 (tension), G8 (uncooperativeness), or G14 (poor impulse control)

- $\geq 25 \%$ increase in PANSS total score (if score at baseline was $>45$ ) or $\geq 10$-point increase in the PANSS total score (if score at baseline was $\leq 45$ ), at two consecutive visits within 7 days

- Increase in a PANSS item score $\geq 5$ (if score at randomization was $\leq 3$ ) for P1, P2, P3, P4, P6, P7, G4, G8, or G14

- Increase in CGI-S-SCA $\geq 2$ points (if score at randomization was 1-3) or $\geq 1$ point (if score at randomization was $\geq 4$ ).

To ensure prominent psychotic and mood symptoms at baseline, subjects had to have a score of $\geq 4$ on three or more of the prespecified PANSS items and YMRS and/or HDRS-21 scores $\geq 16$. No oral supplementation was permitted, but prior antidepressants, mood stabilizers, and benzodiazepines could be continued if at a stable dose; subjects could not be on mood stabilizers and antidepressants together.

Treatment with PLAI significantly delayed time to relapse compared with placebo $(P<0.001)$, and a significantly lower percentage of subjects treated with PLAI (15.2\%) compared with placebo (33.5\%) experienced a relapse event $(P<0.001)$. The relapse risk was 2.49 -fold higher for placebo compared with PLAI (hazard ratio $2.49,95 \%$ confidence interval [CI], 1.55-3.99; $P<0.001)$ in the double-blind phase. The relapse risk was significantly higher for placebo versus PLAI for monotherapy $(P=0.002)$ and for adjunctive therapy $(P=0.021)$, as well as for psychotic $(P<0.001)$, depressive $(P=0.006)$, or manic $(P=0.012)$ relapses. The most common reasons for relapse included worsening of symptoms, interventions to prevent hospitalizations, or the need for additional medications; hospitalization was more frequent for placebo than for PLAI groups (7.1\% and 3.0\%, respectively). For the secondary endpoints, the least-squares mean between-group differences for change was significant for PLAI versus placebo in HDRS-21, YMRS, PANSS, and CGI-S-SCA total scores (all $P<0.001$ ). All-cause discontinuation rate was significantly greater for placebo than for PLAI groups (61.8\% and $39.0 \%$, respectively) $(P<0.001)$. Further, the time to all-cause discontinuation in the double-blind phase was significantly shorter for placebo than for PLAI $(P<0.001)$; the most common reason for discontinuation was withdrawal of consent.

It should be noted that the use of impending relapse criteria allowed for the early recognition and intervention to reduce the risk of a full relapse, which in turn may have reduced the magnitude of change of certain secondary efficacy variables as well as attenuating differences in changes in overall functioning, since patients do not decompensate as much. Nonetheless, this relapse-prevention study demonstrated the efficacy and safety of PLAI whether used as monotherapy or adjunctive therapy in the long-term treatment of SCA. The overall treatment effect was less for adjunctive therapy; this may have reflected a more refractory illness in these subjects and/or the partial efficacy of adjunctive treatment.

\section{Safety and tolerability of PLAI in SCA}

In the relapse-prevention study, the most common ( $\geq 5 \%$ incidence) TEAEs for placebo and PLAI included SCA (5.9\% and $3.0 \%$, respectively), weight increase $(4.7 \%$ 
and $8.5 \%$, respectively), nasopharyngitis (3.5\% and 5.5\%, respectively), headache ( $3.5 \%$ and $5.5 \%$, respectively), and insomnia (7.1\% and $4.9 \%$, respectively).$^{65}$ Most TEAEs were mild to moderate in severity; psychiatric disorder-related events were the most frequent serious TEAEs, which were greater for placebo compared with PLAI (9.4\% and 5.5\%, respectively). It should be noted that the requirement of the 25-week, open-label phase with a stabilization period likely contributed to the selection of patients with good tolerability, but there were fewer subjects who discontinued treatment due to TEAEs with placebo compared with PLAI (1.8\% and $7.3 \%$, respectively). Extrapyramidal symptoms were less frequently reported for placebo than for PLAI $(7.1 \%$ and $8.5 \%$, respectively), but the percentage of subjects receiving antiparkinsonian medications was almost identical (18.8\% and $18.9 \%$, respectively). Prolactin-related TEAEs were less frequently reported with placebo than with PLAI for women (5.8\% and $13.9 \%$, respectively) and men (1.2\% and $7.1 \%$, respectively). Glucose-related TEAEs were more frequently reported with placebo than with PLAI (2.4\% and $1.8 \%$, respectively). Both placebo and PLAI were associated with a slight decrease in mean (standard deviation [SD]) weight $(-0.8[4.5] \mathrm{kg}$ and $-0.2[6.1] \mathrm{kg}$, respectively), while the proportion of subjects with $\mathrm{a} \geq 7 \%$ weight increase was less with placebo than with PLAI $(6.0 \%$ and $13.0 \%$, respectively). The overall TEAE profile of PLAI was consistent with a long-term, relapse-prevention study of similar design in patients with schizophrenia, and there were no new safety signals. ${ }^{66}$ Of interest, weight gain in the relapse-prevention study in schizophrenia was greater at $1.9 \mathrm{~kg}$ for PLAI versus no change for placebo.

\section{Functionality and treatment satisfaction with PLAI in SCA}

Patient functioning in the relapse-prevention study was assessed with the Personal and Social Performance (PSP) scale, which comprises four functional domains (socially useful activities, personal/social relationships, self-care, and disturbing/aggressive behavior). ${ }^{65}$ A significant improvement was observed in the mean change scores at month 15 from baseline for PLAI compared with placebo $(P=0.014)$; the least-squares mean difference was 3.3 (95\% CI, 0.68-5.95). For PLAI, there was an increase in the proportion of patients rated as having good functioning (PSP scores $>70$ ) from $57.9 \%$ to $59.0 \%$ (double-blind baseline to endpoint) compared with a decrease for placebo from $50.6 \%$ to $41.1 \%$ (between-group difference, $P=0.002$ ).

Satisfaction with treatment in the relapse-prevention study was assessed with the Medication Satisfaction
Questionnaire, which is a single-item questionnaire that was originally developed to evaluate satisfaction with antipsychotic medication in patients with schizophrenia. ${ }^{65,67}$ For PLAI, there were significantly more patients $(94.5 \%$ and $85.7 \%$, double-blind baseline and endpoint, respectively) who expressed satisfaction with their treatment on the Medication Satisfaction Questionnaire compared with placebo (93.5\% and 69.6\%, double-blind baseline and endpoint, respectively; between-group difference, $P<0.001$ ).

\section{Place in therapy of PLAl}

SCA is a chronic, severe, and disabling mental illness that has been difficult to define because of symptom overlap with schizophrenia and bipolar disorder and broader heterogeneity. As a consequence, SCA has been less well studied, with the diagnosis being often delayed and ensuing treatment complex, resulting in intermediate outcomes compared to schizophrenia and bipolar disorder. However, the rates of substance use, hospitalization, and suicide are greater than in schizophrenia, while adherence to treatment rates is equally poor. Typical treatment has been empirically based with no widely accepted guidelines of treatment. Nonetheless, the SGAs have been increasingly used in the management of SCA. The first agent to receive formal approval for the treatment of SCA was the oral SGA, paliperidone ER, on the basis of two short-term randomized controlled trials. Two recent expert consensus guidelines recommend the use of LAIs in the management of SCA. While there are no studies of FGA-LAIs in SCA, there has been one long-term study with RLAI in SCA showing significant benefit.

In a long-term, double-blind study, the LAI formulation of paliperidone was shown to significantly delay and reduce relapse compared with placebo. This study demonstrated the efficacy and safety of PLAI when used as either monotherapy or adjunctive therapy for the maintenance treatment of SCA, which represents a population at high risk for poor adherence with oral medications and consequent relapse. A US medication database study found that the mean medication adherence rate was $46 \%$ versus $80 \%$ for the 60 days pre- and postindex hospitalization for SCA, respectively, while the associated costs were US\$2,370 and US\$1,765, respectively $(P<0.001){ }^{68}$ This is particularly important given the high rate of rehospitalization in SCA, which is approximately twice that in schizophrenia. The use of an LAI compared with oral antipsychotics was shown in a Canadian medication database study to reduce hospitalization by approximately half, and to decrease health-related costs from $\$ 24,382$ to $\$ 13,090$, respectively $(P<0.001)$, in patients with schizophrenia and SCA. ${ }^{69}$ 
The efficacy and safety profile of PLAI in patients with SCA is consistent with a similarly designed study conducted in patients with schizophrenia, suggestive of a benefit in the long-term control of not only psychotic but also affective symptoms. Further, as monotherapy, the use of PLAI simplifies treatment by reducing complex pharmacotherapy and eliminating the necessity of daily oral medications. The importance of an individualized treatment plan that incorporates psychoeducation and evidence-based pharmacotherapy while addressing adherence (and treatment satisfaction) is recommended for optimal outcome in SCA. ${ }^{70}$

\section{Conclusion}

In conclusion, despite the dearth of evidence-based data, three, randomized, controlled studies provide evidence of the efficacy of the SGA, paliperidone, in the treatment of SCA. Currently, the two formulations of paliperidone, oral and LAI, represent the only approved treatments for SCA. Further, the use of paliperidone as an LAI formulation represents an additional advantage in terms of improved adherence and simplified treatment in the long-term management of SCA.

\section{Disclosure}

$\mathrm{PC}$ has received research grants and honoraria from Janssen, Pfizer, Eli Lilly, AstraZeneca, Otsuka, GlaxoSmithKline, Lundbeck, Bristol-Myers Squibb, Hoffmann-La Roche, Sunovion, Mylan, Valeant, Paladin, and Novartis. JC has no conflicts of interest to declare. No funding or support from any source was received for the preparation of this article.

\section{References}

1. Nasrallah HA, Goldberg JF, Correll CU; SAD Working Group. Differential diagnosis and therapeutic management of schizoaffective disorder. Ann Clin Psychiatry. 2010;22(suppl 1):S1-S12.

2. Malaspina D, Owen MJ, Heckers S, et al. Schizoaffective disorder in the DSM-5. Schizophr Res. 2013;150(1):21-25.

3. Cheniaux E, Landeira-Fernandez J, Lessa Telles L, et al. Does schizoaffective disorder really exist? A systematic review of the studies that compared schizoaffective disorder with schizophrenia or mood disorders. J Affect Disord. 2008;106(3):209-217.

4. Wilson JE, Nian H, Heckers S. The schizoaffective disorder diagnosis: a conundrum in the clinical setting. Eur Arch Psychiatry Clin Neurosci. 2014;264(1):29-34.

5. American Psychiatric Association. Diagnostic and Statistical Manual of Mental Disorders. 5th ed. Arlington, VA: American Psychiatric Publishing; 2013.

6. Murru A, Pacchiarotti I, Nivoli AM, et al. Is schizoaffective disorder still a neglected condition in the scientific literature? Psychother Psychosom. 2012;81(6):389-390.

7. Perälä J, Suvisaari J, Saarni SI, et al. Lifetime prevalence of psychotic and bipolar I disorders in a general population. Arch Gen Psychiatry. 2007;64(1):19-28.

8. Bromet EJ, Kotov R, Fochtmann LJ, et al. Diagnostic shifts during the decade following first admission for psychosis. Am J Psychiatry. 2011; 168(11):1186-1194.
9. Salvatore P, Baldessarini RJ, Tohen M, et al. McLean-Harvard International First-Episode Project: two-year stability of DSM-IV diagnoses in 500 first-episode psychotic disorder patients. J Clin Psychiatry. 2009;70(4):458-466.

10. Harrow M, Grossman LS, Herbener ES, et al. Ten-year outcome: patients with schizoaffective disorders, schizophrenia, affective disorders and mood-incongruent psychotic symptoms. Br J Psychiatry. 2000;177:421-426.

11. Chang CK, Hayes RD, Perera G, et al. Life expectancy at birth for people with serious mental illness and other major disorders from a secondary mental health care case register in London. PLoS One. 2011; 6(5):e19590.

12. Canuso CM, Kosik-Gonzalez C, Sheehan J, et al. Frequency of schizoaffective disorder in an International patient population with psychotic disorders using the mini-international neuropsychiatric interview. Schizophr Res. 2010;118(1-3):305-306.

13. Kent S, Fogarty M, Yellowlees P. Heavy utilization of inpatient and outpatient services in a public mental health service. Psychiatr Serv. 1995; 46(12):1254-1257.

14. Altamura AC, Mundo E, Bassetti R, et al. Transcultural differences in suicide attempters: analysis on a high-risk population of patients with schizophrenia or schizoaffective disorder. Schizophr Res. 2007; 89(1-3):140-146.

15. Bhatia T, Thomas P, Semwal P, et al. Differing correlates for suicide attempts among patients with schizophrenia or schizoaffective disorder in India and USA. Schizophr Res. 2006;86(1-3):208-214.

16. Hor K, Taylor M. Suicide and schizophrenia: a systematic review of rates and risk factors. $J$ Psychopharmacol. 2010;24(4 suppl):81-90.

17. Drake RJ. Insight into illness: impact on diagnosis and outcome of nonaffective psychosis. Curr Psychiatry Rep. 2008;10(3):210-216.

18. Bora E, Yucel M, Pantelis C. Cognitive functioning in schizophrenia, schizoaffective disorder and affective psychoses: meta-analytic study. Br J Psychiatry. 2009;195(6):475-482.

19. Lindenmayer JP, Kay SR, Van Praag H. Distinction of schizoaffective from profile. Independent validation. Schizophr Res. 1989;2(4-5):423-424.

20. Marneros A, Deister A, Rohde A. Psychopathological and social status of patients with affective, schizophrenic and schizoaffective disorders after long-term course. Acta Psychiatr Scand. 1990;82(5):352-358.

21. Lysaker PH, Davis LW. Social function in schizophrenia and schizoaffective disorder: associations with personality, symptoms and neurocognition. Health Qual Life Outcomes. 2004;2:15.

22. Martín-Subero M, Berk L, Dodd S, et al. Quality of life in bipolar and schizoaffective disorder - a naturalistic approach. Compr Psychiatry. 2014;55(7):1540-1545.

23. Saarni SI, Viertiö S, Perälä J, et al. Quality of life of people with schizophrenia, bipolar disorder and other psychotic disorders. Br J Psychiatry. 2010;197(5):386-394.

24. Laursen TM, Labouriau R, Licht RW, et al. Family history of psychiatric illness as a risk factor for schizoaffective disorder: a Danish registerbased cohort study. Arch Gen Psychiatry. 2005;62(8):841-848.

25. Potash JB. Carving chaos: genetics and the classification of mood and psychotic syndromes. Harv Rev Psychiatry. 2006;14(2):47-63.

26. Cosgrove VE, Suppes T. Informing DSM-5: biological boundaries between bipolar I disorder, schizoaffective disorder, and schizophrenia. BMC Med. 2013;11:127.

27. Glausier JR, Kimoto S, Fish KN, et al. Lower glutamic acid decarboxylase $65-\mathrm{kDa}$ isoform messenger RNA and protein levels in the prefrontal cortex in schizoaffective disorder but not schizophrenia. Biol Psychiatry. 2015;77(2):167-176.

28. Morris HM, Hashimoto T, Lewis DA. Alterations in somatostatin mRNA expression in the dorsolateral prefrontal cortex of subjects with schizophrenia or schizoaffective disorder. Cereb Cortex. 2008; 18(7):1575-1587.

29. Morris HM, Stopczynski RE, Lewis DA. NPY mRNA expression in the prefrontal cortex: selective reduction in the superficial white matter of subjects with schizoaffective disorder. Schizophr Res. 2009; 115(2-3):261-269. 
30. Bulbul F, Virit O, Alpak G, et al. Are oxidative stress markers useful to distinguish schizoaffective disorder from schizophrenia and bipolar disorder? Acta Neuropsychiatr. 2014;26(2):120-124.

31. Olfson M, Marcus SC, Wan GJ. Treatment patterns for schizoaffective disorder and schizophrenia among Medicaid patients. Psychiatr Serv. 2009;60(2):210-216.

32. Malhi GS, Green M, Fagiolini A, et al. Schizoaffective disorder: diagnostic issues and future recommendations. Bipolar Disord. 2008; 10(1 pt 2):215-230.

33. Murru A, Pacchiarotti I, Nivoli AM, et al. What we know and what we don't know about the treatment of schizoaffective disorder. Eur Neuropsychopharmacol. 2011;21(9):680-690.

34. McElroy SL, Keck PE Jr, Strakowski SM. An overview of the treatment of schizoaffective disorder. J Clin Psychiatry. 1999;60(suppl 5): $16-21$.

35. Flynn J, Grieger TA, Benedek DM. Pharmacologic treatment of hospitalized patients with schizoaffective disorder. Psychiatr Serv. 2002; 53(1):94-96.

36. Lerner V, Libov I, Kotler M, et al. Combination of "atypical" antipsychotic medication in the management of treatment-resistant schizophrenia and schizoaffective disorder. Prog Neuropsychopharmacol Biol Psychiatry. 2004;28(1):89-98.

37. Levinson DF, Umapathy C, Musthaq M. Treatment of schizoaffective disorder and schizophrenia with mood symptoms. Am J Psychiatry. 1999;156(8):1138-1148.

38. Murru A, Pacchiarotti I, Nivoli AM, et al. Rates and clinical correlates of treatment non-adherence in schizoaffective bipolar patients. Acta Psychiatr Scand. 2012;125(5):412-418.

39. Murru A, Pacchiarotti I, Amann BL, et al. Treatment adherence in bipolar I and schizoaffective disorder, bipolar type. J Affect Disord. 2013; 151(3):1003-1008.

40. Bodén R, Brandt L, Kieler H, et al. Early non-adherence to medication and other risk factors for rehospitalization in schizophrenia and schizoaffective disorder. Schizophr Res. 2011;133(1-3):36-41.

41. Lindenmayer JP, Liu-Seifert H, Kulkarni PM, et al. Medication nonadherence and treatment outcome in patients with schizophrenia or schizoaffective disorder with suboptimal prior response. J Clin Psychiatry. 2009;70(7):990-996.

42. Cascade E, Kalali AH, Buckley P. Treatment of schizoaffective disorder. Psychiatry (Edgmont). 2009;6(3):15-17.

43. Keck PE Jr, McElroy SL, Strakowski SM. Schizoaffective disorder: role of atypical antipsychotics. Schizophr Res. 1999;35(suppl):S5-S12.

44. Vieta E, Herraiz M, Fernández A, et al. Efficacy and safety of risperidone in the treatment of schizoaffective disorder: initial results from a large, multicenter surveillance study. Group for the Study of Risperidone in Affective Disorders (GSRAD). J Clin Psychiatry. 2001; 62(8):623-630.

45. Murru A, Hidalgo D, Bernardo M. Antipsychotic switching in schizoaffective disorder: a systematic review. World J Biol Psychiatry. 2015:1-19.

46. Risperdal $®$ Consta $®$ Risperidone Long-Acting Injection (Prescribing Information). Titusville, NJ: Janssen Pharmaceuticals, Inc.; 2007. [Revised 2014].

47. Mohl A, Westlye K, Opjordsmoen S, et al. Long-acting risperidone in stable patients with schizoaffective disorder. J Psychopharmacol. 2005; 19(5 suppl):22-31.

48. McDonnell DP, Landry J, Detke HC. Long-term safety and efficacy of olanzapine-acting injection in patients with schizophrenia or schizoaffective disorder: a 6-year, multinational, single-arm, open-label study. Int Clin Psychopharmacol. 2014;29(6):322-331.

49. Chue PS, MacKenzie EM, Chue JA, et al. The pharmacology and formulation of paliperidone extended release. Expert Rev Neurother. 2012; 12(12):1399-1410.

50. Invega ${ }^{\circledR}$ Paliperidone Extended-Release Tablets (Prescribing Information). Titusville, NJ: Janssen Pharmaceuticals, Inc.; 2007. [Revised 2014].
51. Canuso CM, Turkoz I, Fu DJ, et al. Role of paliperidone extended-release in treatment of schizoaffective disorder. Neuropsychiatr Dis Treat. 2010;6:667-679.

52. Yang LP. Oral paliperidone: a review of its use in the management of schizoaffective disorder. CNS Drugs. 2011;25(6):523-538.

53. Canuso CM, Battisti WP. Paliperidone extended-release: a review of efficacy and tolerability in schizophrenia, schizoaffective disorder and bipolar mania. Expert Opin Pharmacother. 2010;11(15):2557-2567.

54. Invega Sustenna ${ }^{\circledR}$ Paliperidone Palmitate Extended-Release Injectable Suspension (Prescribing Information). Titusville, NJ: Janssen Pharmaceuticals, Inc.; 2009. [Revised 2015].

55. Haddad $P$, Lambert T, Lauriello J. The role of antipsychotic long-acting injections in current practice. In: Haddad P, Lambert T, Lauriello J, editors. Antipsychotic Long-acting Injections. Oxford: Oxford University Press; 2011:241-256.

56. Chou YH, Chu PC, Wu SW, et al. A systemic review and experts' consensus for long-acting injectable antipsychotics in bipolar disorder. Clin Psychopharmacol Neurosci. 2015;13(2):121-128.

57. Llorca PM, Abbar M, Courtet P, et al. Guidelines for the use and management of long-acting injectable antipsychotics in serious mental illness. BMC Psychiatry. 2013;13:340.

58. Corena-McLeod M. Comparative pharmacology of risperidone and paliperidone. Drugs R D. 2015;15(2):163-174.

59. Álamo C, López-Muñoz F. The pharmacological role and clinical applications of antipsychotics' active metabolites: paliperidone versus risperidone. Clin Exp Pharmacol. 2013;3:1-117.

60. Clarke WP, Chavera TA, Silva M, et al. Signalling profile differences: paliperidone versus risperidone. Br J Pharmacol. 2013;170(3): 532-545.

61. Chue P, Chue J. A review of paliperidone palmitate. Expert Rev Neurother. 2012;12(12):1383-1397.

62. Gilday E, Nasrallah HA. Clinical pharmacology of paliperidone palmitate a parenteral long-acting formulation for the treatment of schizophrenia. Rev Recent Clin Trials. 2012;7(1):2-9.

63. Canuso CM, Schooler N, Carothers J, et al. Paliperidone extendedrelease in schizoaffective disorder: a randomized, controlled study comparing a flexible dose with placebo in patients treated with and without antidepressants and/or mood stabilizers. J Clin Psychopharmacol. 2010;30(5):487-495.

64. Canuso CM, Lindenmayer JP, Kosik-Gonzalez C, et al. A randomized, double-blind, placebo-controlled study of 2 dose ranges of paliperidone extended-release in the treatment of subjects with schizoaffective disorder. J Clin Psychiatry. 2010;71(5):587-598.

65. Fu DJ, Turkoz I, Simonson RB, et al. Paliperidone palmitate oncemonthly reduces risk of relapse of psychotic, depressive, and manic symptoms and maintains functioning in a double-blind, randomized study of schizoaffective disorder. J Clin Psychiatry. 2015;76(3):253-262.

66. Hough D, Gopal S, Vijapurkar U, et al. Paliperidone palmitate maintenance treatment in delaying the time-to-relapse in patients with schizophrenia: a randomized, double-blind, placebo-controlled study. Schizophr Res. 2010;116(2-3):107-117.

67. Vernon MK, Revicki DA, Awad AG, et al. Psychometric evaluation of the Medication Satisfaction Questionnaire (MSQ) to assess satisfaction with antipsychotic medication among schizophrenia patients. Schizophr Res. 2010;118(1-3):271-278.

68. Karve S, Markowitz M, Fu DJ, et al. Assessing medication adherence and healthcare utilization and cost patterns among hospital-discharged patients with schizoaffective disorder. Appl Health Econ Health Policy. 2014;12(3):335-346.

69. Lachaine J, Lapierre ME, Abdalla N, et al. Impact of switching to longacting injectable antipsychotics on health services use in the treatment of schizophrenia. Can J Psychiatry. 2015;60(3 supp1 2):S40-S47.

70. Vieta E. Developing an individualized treatment plan for patients with schizoaffective disorder: from pharmacotherapy to psychoeducation. J Clin Psychiatry. 2010;71(suppl 2):14-19. 


\section{Publish your work in this journal}

Therapeutics and Clinical Risk Management is an international, peerreviewed journal of clinical therapeutics and risk management, focusing on concise rapid reporting of clinical studies in all therapeutic areas, outcomes, safety, and programs for the effective, safe, and sustained use of medicines. This journal is indexed on PubMed Central, CAS,

EMBase, Scopus and the Elsevier Bibliographic databases. The manuscript management system is completely online and includes a very quick and fair peer-review system, which is all easy to use. Visit http://www.dovepress.com/testimonials.php to read real quotes from published authors.

Submit your manuscript here: http://www.dovepress.com/therapeutics-and-clinical-risk-management-journal 